\title{
CONDUCTIVITY AND MAGNETISM OF MAGNETIC OXIDES
}

\author{
G.A. Gehring \\ Department of Physics and Astronomy, University of Sheffield \\ Sheffield S3 7RH, U.K.
}

\begin{abstract}
In a stoichiometric oxide the energy for the magnetic ordering is due to superexchange. This depends on the virtual transfer of a $d$ electron from the transition ion to the neighbouring oxygen. When the oxide is $p$-doped there are compensating holes on the oxygen or the transition ion becomes mixed valent. The oxide may then conduct. The same transfer integral enters both the expression for the antiferromagnetic superexchange and the band width of the mobile carriers. Thus materials with a large antiferromagnetic exchange energy will be expected to have a relatively wide conduction band in the doped state and hence to have a high conductivity. In this paper the difference is explored between the materials in which there is true antiferromagnetism and those which are ferrimagnetic. In the antiferromagnets the carriers must destroy the magnetic order as they move. This behaviour is well known from the cuprates. In ferrimagnets the carriers may be able to move entirely on one sublattice. This occurs in $\mathrm{Fe}_{3} \mathrm{O}_{4}$ and probably in the doped garnets. In the case where motion is on one sublattice then doping does not destroy the magnetism and there is a relatively small magnetoresistance. An interesting feature is that unlike the cuprates the ferrimagnets do not become good metals at any doping but exhibit hopping conductivity. We explain the apparent paradox that the best conductivity is actually observed in materials where the conduction is only allowed when the antiferromagnetism has been quenched and that the conductivity in ferrimagnets is low.
\end{abstract}

PACS numbers: $75.50 . \mathrm{Pp}, 75.50 . \mathrm{Gg}$

\section{Introduction}

In this paper we review the competition between antiferromagnetic order and electrical conductivity. This occurs whether or not the undoped material is a Mott-Hubbard or charge transfer insulator. In the doped state the transition ion becomes mixed valent in the former case and in the latter the holes are predominantly on the oxygen. The same transfer integral enters both the expression for the antiferromagnetic superexchange and the band width of the mobile carriers. Thus materials with a large antiferromagnetic exchange energy will be expected 
to have a relatively wide conduction band in the doped state and hence to have a high conductivity.

There is an interesting contrast between materials which are antiferromagnetic and those which are ferrimagnetic. In the antiferromagnets the conductivity can only occur after the long range antiferromagnetic order is quenched. Any residual antiferromagnetic correlations might be expected to impede the conductivity. In ferrimagnets the conductivity may (and usually does) occur on one sublattice where the spins are already held parallel by the exchange interactions. Thus in this case the additional tendency to support ferromagnetism from the conductivity only strengthens the relevant exchange interactions. However in these materials the band widths are so narrow that the addition of disorder and polaronic interactions cause the materials to show hopping conductivity. We have the paradoxical situation that the materials in which the conductivity must compete with the magnetism become the best conductors while those for which the magnetism and the conductivity coexist are not metallic.

A further interesting case is given by the manganites where the underlying antiferromagnetic structure ( $A$ type) would allow nearest neighbour charge transport in the ferromagnetic planes which would not destroy the antiferromagnetism. This does not occur. The charges move in all three directions, the antiferromagnetism is rapidly quenched and a ferromagnetic metallic phase occurs.

In this article we first review the theory of conductivity in antiferromagnets and then discuss the properties of four representative materials. Two antiferromagnets, a doped cuprate and a manganite, and two ferrimagnets, magnetite and a doped garnet. Finally we discuss why the ferrimagnets show such poor conductivity at low temperatures. We speculate that this is not a coincidence but is likely to be characteristic of all ferrimagnets.

\section{Conductivity in antiferromagnets}

It has been known for a long time that a charge cannot propagate freely in a Hubbard antiferromagnet $[1,2]$. The charge transfer integral depends on the overlap of the spatial wave functions and so conserves spin. We consider the situation of a simple antiferromagnet with an electron missing on one site. In Fig. 1 we consider the case of $S=1 / 2$ and in Fig. $2, S>1 / 2$. In both cases a single electron and a spin $\downarrow$ have been removed from the central site. We first consider the case of $S=1 / 2$ in configuration shown in Fig. 1a. One of the up spin electrons from the outer part of the cluster can hop in to the central site as shown in Fig. 1b however in the two-dimensional case shown such a hop will bring an up spin into a position where it is parallel to three neighbours. Such a transition is to a state with a high exchange energy. It can be shown that all subsequent hops of a single hole only add to the exchange energy. Hence such motion is strongly inhibited by antiferromagnetism. This argument was quasi-classical as no spin exchange was included but the inclusion of full quantum effects does not affect these conclusions.

However if the spins became parallel in the neighbourhood of the charge then the spin can move freely in a ferromagnetic background and so lower its kinetic energy. In the limit of the ratio $J / t \rightarrow 0$ then it was shown that if the ground 
a
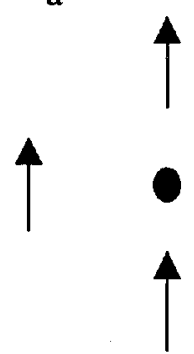

b
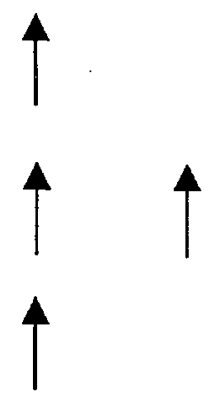

Fig. 1. (a) A hole on a "down spin" site in a $\mathrm{Cu}-\mathrm{O}$ plane; (b) the same configuration after the hole has made one hop.

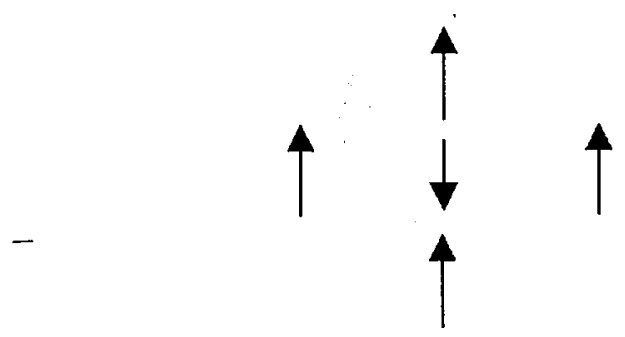

Fig. 2. A hole of spin $1 / 2$ on a "down spin" site in an antiferromagnet with $S>1 / 2$. The central site has spin $S$ and the outer sites have total spin $S+1 / 2$.

state is a spin $1 / 2$ antiferromagnet in the absence of a doping then a single hole will stabilise a ferromagnetic ground state. This is the Nagaoka theorem [2].

Since the discovery of the behaviour of the doped cuprates there has been much progress in understanding the effects of doping antiferromagnets. It was realised very early that the Nagaoka theorem is valid only for one hole. The interaction of many mobile holes in a material with antiferromagnetic coupling is a very difficult problem (which is well addressed by other papers in this volume). However the result that conductivity is only possible in the absence of long range antiferromagnetic order is well established.

The situation.shown in Fig. 2 is different because we must consider the Hund rule coupling on all sites. A different result is obtained from classical and quantum mechanical treatments.

The surrounding spins are assumed to have both total spin and $z$ component of total spin, $S_{\mathrm{T}}$, equal to $S+1 / 2$. We remove one $S=1 / 2$ from one of the outer sites and transfer it to the central site. We assume strong Hund's rule complying so that the state with $S_{\mathrm{T}}=S-1 / 2$ on the central site has an energy which is higher by the intra-atomic Hund rule exchange energy.

We consider the classical case first [1]. In this approximation the background spins $S$ are fixed so in order to obtain the state of total spin $S_{\mathrm{T}}$ of $S+1 / 2$ we need to project the spinor on to the new axis and the net transfer integral is modified from $t$ to $t \cos \theta / 2$. (The half angle arises because of the transformation properties 
of a spinor wave function.) This takes the maximum value of $t$ for $\theta=0$ but vanishes for the antiparallel case of $\theta=\pi$. We compare the energy to move one hole in a classical antiferromaget for $S=1 / 2$ and $S>1 / 2$. In both cases the movement of charge is inhibited by the inter-atomic exchange but in the case of $S>1 / 2$ the stronger intra-atomic exchange also opposes the motion.

In the quantum treatment we need to consider the projection of the state formed by the direct product of $|S: M\rangle$ and $|1 / 21 / 2\rangle$ on to the state $\mid S+1 / 2$, $M+1 / 2\rangle$. This was shown by Kubo and Ohata [3] to be proportional to $\sqrt{\frac{S+M+1}{2 S+1}}$. In the case where $M=S$ the result is unity which is identical with the classical result with $\theta=0$ but the quantum result differs from the classical case of $\theta=\pi$ most strongly. In the quantum treatment for antiparallel alignment $M=-S$ for which the charge transfer is proportional to $\frac{1}{\sqrt{2 S+1}}$ which does not vanish except in the limit of infinite spin.

However the charge transfer is strongest for the ferromagnetic alignment so giving the largest energy reduction due to the hole band width. So once again charge transfer induces ferromagnetism. This is known as Zener double exchange [4]. In the ferromagnetic state the holes propagate freely and fill the lowest band states. The consequent reduction in energy of the whole lattice is a measure of the strength of the double exchange.

\section{Case histories}

In this section we consider four representative materials. All are either intrinsically conducting or can be doped. Two are antiferromagnets in the undoped phase and two are ferrimagnetic.

\section{1. $\mathrm{La}_{2} \mathrm{CuO}_{4}$ doped to $\mathrm{La}_{2-x} \mathrm{Sr}_{x} \mathrm{CuO}_{4}$}

In this material the copper and oxygen ions lie on planes. The $S=1 / 2$ spins on the $\mathrm{Cu}$ ions are due to one hole in a non-degenerate $\left\{x^{2}-y^{2}\right\} d$ orbital and order antiferromagnetically when it is undoped. The Néel temperature of $300 \mathrm{~K}$ arises from strong exchange interactions in the planes and has been reduced because of the quasi-two-dimensional nature of the lattice. The long range antiferromagnetic order disappears very rapidly when the planes are doped with holes vanishing by about $6 \%$. This arises because the holes are so mobile so that each mobile hole can destroy the antiferromagnetic order associated with many $\mathrm{Cu}$ sites.

As the cuprate is doped it is conducting and then superconducting. The conducting phase is highly anomalous as the resistivity varies linearly with temperature. Although the long range antiferromagnetism has disappeared there is still evidence of short range, long-lived, antiferromagnetic correlations from neutron scattering [5] and muon spin resonance [6].

The interesting point for this discussion is the magnitude of the conductivity. It is certainly in the metallic range. Thus the presence of the spin correlations is not causing strong scattering as they are incorporated into some sort of coherent quantum state which may be a marginal Fermi liquid [7]. 


\section{2. $\mathrm{LaMnO}_{3}$ doped to $\mathrm{La}_{1-x} \mathrm{Sr}_{x} \mathrm{MnO}_{3}$}

This compound is one of a family of compounds with different trivalent elements replacing $\mathrm{La}$ and different divalent elements replacing $\mathrm{Sr}$ which have become known as the CMR (colossal magnetoresistance) materials. It is a manganite. The interesting region is around $x=0.3$ where the CMR effects are seen. The physical properties of the materials and the CMR effect have been well reviewed [8,9].

In $\mathrm{LaMnO}_{3}$ the $\mathrm{Mn}$ ions are in the valence state $\mathrm{Mn}^{3+}$ with where the four $d$ electrons have parallel spin due to Hund's rule coupling. Three of them are in the $t_{2 \mathrm{~g}}$ orbitals and the fourth is in the (upper) $e_{\mathrm{g}}$ orbital. The $e_{\mathrm{g}}$ orbital is strongly Jahn-Teller active so the crystal is distorted to become tetragonal and the degeneracy of the $e_{\mathrm{g}}$ state is split. The net result is that the crystal forms an $A$-type antiferromagnetic structure in which $x-y$ planes of spins are coupled ferromagnetically and the planes are stacked antiferromagnetically up the $z$ axis which is also the $c$ axis of the tetragonal structure.

As the material is doped with a divalent ion at around $x=0.3$ it has a low temperature transition to a ferromagnetic ground state because of the Zener exchange as described in Sec. 1. In the high temperature phase the resistivity shows typical activation behaviour leading to a maximum in the resistivity near to $T_{\mathrm{c}}$. This maximum is suppressed in a magnetic field leading to colossal magnetoresistance.

In the low temperature phase the tendency towards antiferromagnetism at low doping causes very large resistivities to occur at grain boundaries. A typical range of conductivity at low temperature is given in Table. We note that these conductivities are rather lower than the cuprates but still metallic.

TABLE

A summary of the physical properties of the material discussed in this article.

\begin{tabular}{c|l|c|c|c}
\hline \hline Compound & \multicolumn{1}{|c|}{ Notes } & $T_{\mathrm{N}}$ & $\begin{array}{c}\text { Relevant } \\
\text { exchange } \\
\text { parameter }\end{array}$ & $\begin{array}{c}\text { Typical } \rho \\
\text { (resistance) }\end{array}$ \\
\hline $\mathrm{La}_{2-x} \mathrm{Sr}_{x} \mathrm{CuO}_{4}$ & $\begin{array}{l}\text { magnetic lattice } \sim 2 d . \text { An- } \\
\text { tiferrocorrelations persist } \\
\text { into conducting phase } \\
3 d \text { material has Zener ex- }\end{array}$ & $300 \mathrm{~K}$ & $1500 \mathrm{~K}$ & $10^{-4} \Omega \mathrm{cm}$ \\
$\mathrm{La}_{1-x} \mathrm{Sr}_{x} \mathrm{MnO}_{3}$ & $\begin{array}{l}\text { change, CMR } \\
\text { mixed valent on } B \text { sub- } \\
\text { lattice, small Zener com- } \\
\text { pared to } J_{B B} \\
\text { ferrimagnet }\end{array}$ & $835 \mathrm{~K}$ & $3 \mathrm{~K}\left(J_{B B}\right)$ & $10^{-1} \Omega \mathrm{cm}$ \\
$\mathrm{Fe}_{3} \mathrm{O}_{4}$ & $560 \mathrm{~K}$ & $13.4\left(J_{d d}\right)$ & $10^{2}-10^{9}$ \\
$\mathrm{YIG}$ & $\Omega \mathrm{cm}$
\end{tabular}




\section{3. $\mathrm{Fe}_{3} \mathrm{O}_{4}$}

The oldest known magnetic mineral has been heralded as one of the most promising candidates to produce spin polarised carriers for spin electronics. The main reason why it appeared to be so attractive was its high transition temperature of $835 \mathrm{~K}$ which means that even room temperature is well below $T_{\mathrm{c}}$.

It is a ferrimagnet. The $\mathrm{Fe}$ ions on the $A$ sites are all $\mathrm{Fe}^{3+}$, each carries a spin $5 / 2$, and these form the minority sublattice. Half the Fe ions on the two $B$ sites of the majority sublattice are also $\mathrm{Fe}^{3+}$ and have spin $5 / 2$ and the other half are $\mathrm{Fe}^{2+}$ so they have an extra electron which reduces the total spin to $S=2$. The energy level structure is sketched in Fig. 3.

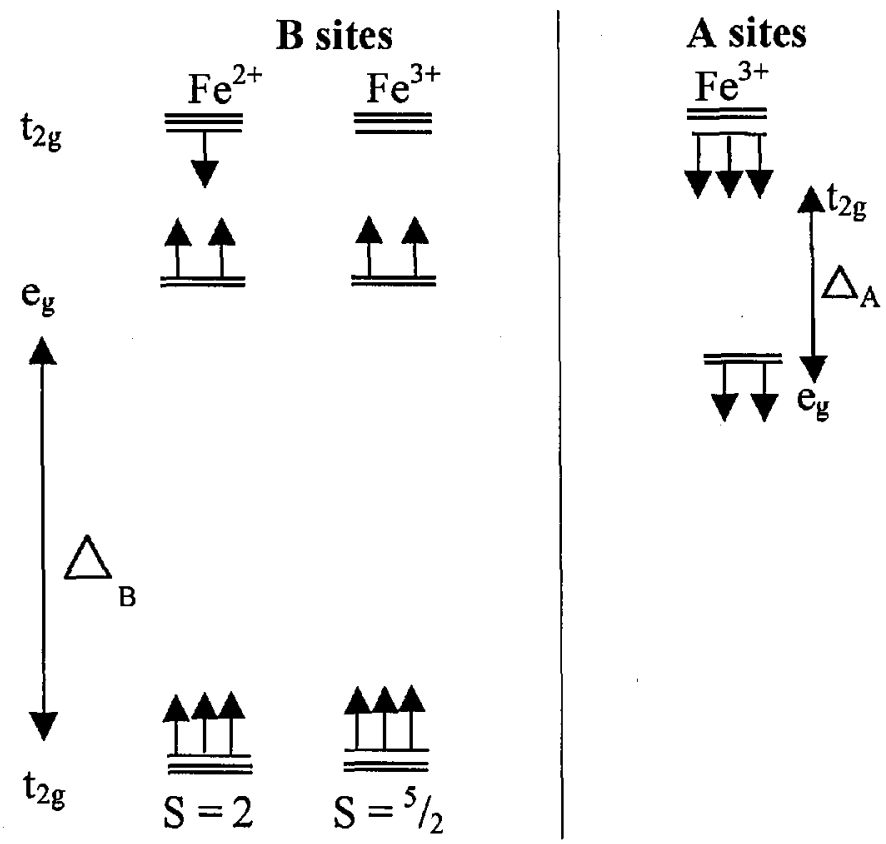

Fig. 3. The energy level scheme for $\mathrm{Fe}_{3} \mathrm{O}_{4}$.

The ferrimagnetic order is stabilised by the following exchange interactions: $J_{A B}=-22 \mathrm{~K}, J_{A A}=-11 \mathrm{~K}, J_{B B}=3 \mathrm{~K}$. The small (and positive) value of $J_{B B}$ is due partly to the fact that the superexchange path is through a $90^{\circ}$ oxygen bond and due to a small amount of Zener double exchange. We note that the double exchange mechanism is slightly different here. The $B$ site ions have their full complement of up spins. Hence the Pauli principle only allows the transfer of a down spin. In the normal Zener exchange discussed earlier the charge may transfer to create a total spin state of $S+1 / 2$; the state $S_{\mathrm{T}}=S-1 / 2$ is allowed but is disfavoured by the Hund rule coupling.

The mobility of this "extra" electron drops dramatically at the Vervey temperature $T_{\mathrm{V}} \approx 120 \mathrm{~K}$ where some kind of "charge freezing" takes place. A typical 
value of the resistance is included in Table. Our concern is with the behaviour at room temperature which is well above $T_{\mathrm{V}}$. In this region the conductivity is low and the temperature dependence is characteristic of polaron or variable range hopping [10].

The ideal picture as found, for example, in band structure studies [11] is that the majority spin band lies entirely below the Fermi level but passes through the minority band formed from the $t_{2 \mathrm{~g}}$ orbitals on the $B$ sites. The band has a rather low occupation as there is one extra electron per two $B$ sites and there are three $t_{2 \mathrm{~g}}$ orbitals per site. Band calculations reveal a band width of $\approx 2 \mathrm{eV}$. An estimate of the Hubbard $U$ gives approximately $4 \mathrm{eV}$ which is large enough to suppress any double occupancy of the $t_{2 \mathrm{~g}}$ orbitals. There is also a strong Coulomb interaction between the occupancy of the $t_{2 \mathrm{~g}}$ orbitals on neighbouring sites. It is believed to be this interaction which drives the Vervey transition [12].

$$
\text { 3.4. } \mathrm{Y}_{3} \mathrm{Fe}_{5} \mathrm{O}_{16} \text { doped to }\left(\mathrm{Y}_{1-x} \mathrm{Ca}_{x}\right)_{3} \mathrm{Fe}_{5} \mathrm{O}_{16}
$$

Yttrium iron garnet (YIG) is a ferrimagnet with an ordering temperature of $560 \mathrm{~K}$. Again we have an ordering temperature which is comfortably above room temperature. Each cell contains five $\mathrm{Fe}^{3+}$ ions all carrying $S=5 / 2$. The five iron sites divide into two groups: the two " $a$ " site ions have octahedral co-ordination, are parallel to each other and form the minority sublattice and the three ions on the tetrahedral " $d$ " sublattice are also parallel to each other and form the majority sublattice. An energy level diagram is sketched in Fig. 4, the ordering of the states follows Larsen and Metselaar [13]. The exchange interactions are $J_{a d}=-39.8 \mathrm{~K}$, $J_{d d}=-13.4 \mathrm{~K}$, and $J_{a a}=-3.8 \mathrm{~K}$.

When YIG is doped with divalent calcium, compensating holes appear on the oxygen ions adjacent to the Fe on the tetrahedral sites [14]. Doping levels up

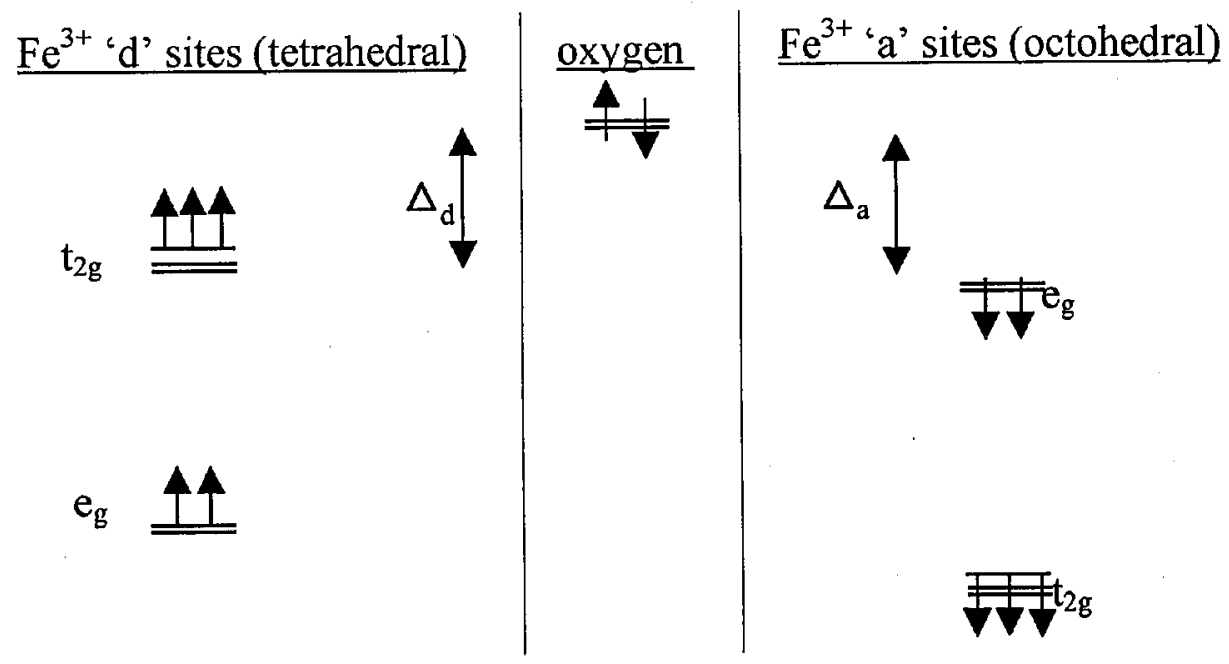

Fig. 4. The energy level scheme for YIG. 
to $20 \%$ have been achieved. The transition temperature at this doping is reduced by only about $5 \%$ and the saturation magnetisation is also little affected.

The geometry of the lattice is such that a hole on an oxygen around a given $d$ site Fe can only hop to a neighbouring cluster via an oxygen-oxygen bond [14]. This relativity weak transfer is responsible for the relatively small value for $J_{d d}$. The conductivity is thermally activated below room temperature with an activation energy of approximately $0.3 \mathrm{eV}$.

\section{Antiferromagnets and ferrimagnets}

When we survey the compounds discussed here we see there are three ways in which they can be classified: as Mott-Hubbard or charge transfer integrals, whether they have spin equal to $1 / 2$ (no Zener exchange) or whether they are antiferromagnets or ferrimagnets. We argue that it is the third difference which is crucial.

The difference between the Mott-Hubbard and the charge transfer compounds is that when they are doped in the former case it is the transition metal ion has a fluctuating valence and in the latter case the compensating holes are on the oxygen. In any real system this is likely to be a matter of degree but some of these materials do fall very clearly into one of the categories. In the cuprates the holes are on the oxygen and this is now believed to be the case also in the manganites [15] and probably the garnets. In magnetite it is believed that it is the $\mathrm{Fe}$ sites which are mixed valent. The reason that this distinction is not really crucial was explained by Zhang and Rice [16]. They showed that a hole doped around a spin $1 / 2$ would bind in a singlet so that the whole cluster appeared as if it were the transition metal ion that had lost its charge and spin. A similar argument has been made for the higher spin cases [17]. Thus this difference between magnetite and the other compounds although real does not seem to be important.

Another difference between the compounds listed here is that the cuprate has spin $1 / 2$ and so cannot exhibit Zener exchange. This is obviously vital in causing the low temperature ferromagnetism in the manganites but cannot account for the large difference in the conductivity between the cuprate and manganite on one hand and magnetite and doped garnets on the other.

In the antiferromagnets we see that the long range antiferromagnetic order is destroyed by even low levels of doping $\approx 5 \%$. This occurs in both the spin- $1 / 2$ system $\mathrm{La}_{2} \mathrm{CuO}_{4}$ in which "wrong bands" are formed by moving holes and in the $S>1 / 2$ systems, such as $\mathrm{LaMnO}_{3}$, in which there is Zener double exchange. In the cuprates the simple antiferromagnetic structure means that any nearest neighbour hopping of the hole necessarily destroys antiferromagnetism. In the doped manganites, $\mathrm{LaMnO}_{3}$, a charge could in principle move in the ferromagnetic $x-y$ planes without destroying the antiferromagnetic order.

By contrast in the ferrimagnets there can be large concentrations of mobile carriers without disrupting the magnetisation. In $\mathrm{Fe}_{3} \mathrm{O}_{4}$ half of the $\mathrm{Fe}$ ions on the $B$ sublattice are in the state $\mathrm{Fe}^{2+}$ so the doping concentrations on that sublattice is $50 \%$; alternatively in the whole unit cell the doping is $33 \%$. Similarly in the garnets high doping levels are achieved for a calcium concentration of $20 \%$ on the $\mathrm{Y}$ sites. There are three $\mathrm{Y}$ sites and three Fe " $d$ " sites in each cell so this again represents 
a relatively high doping of approximately $20 \%$ of one hole being adjacent to each $\mathrm{Fe}$ ion.

Thus there is a marked contrast between the ferrimagnets and antiferromagnets. In the ferrimagnets the charges move on one sublattice only. The spins on a given sublattice are all parallel so that magnetic order assists rather than impedes transport. This explains why the transition temperature is changed rather little. with doping in the garnets. These differences can be readily understood when it is realised that ferrimagnets conduct on one sublattice only.

However one puzzle remains. We have seen that the antiferromagnetic order is suppressed by mobile carriers. We know that there are residual antiferromagnetic correlations in the cuprates so we might expect that the conductivity in the doped antiferromagnets would always be less than that in the ferrimagnets where no such competition between magnetism and conductivity occurs. It is obvious from Table that the reverse is the case. This puzzle can be resolved when it is realised that in the ferrimagnets the intra-sublattice exchange which depends on the inter-sublattice hopping energy is small. This gives rise to band widths that are so narrow that localisation occurs.

The final issue is why is conductivity restricted to a single sublattice in a ferrimagnet; or what is it that determines the fact that charge transfer does not occur between the sublattices in a ferrimagnet so breaking down the ferrimagnetic order? This can be understood immediately by realising that the two sublattices must be chemically inequivalent. Thus the energy states on the two sites will be different and charge transfer will only occur between them to an appreciable extent if the intersublattice hopping is sufficiently large. This does not occur on the systems discussed here. Conversely in the manganites there is no chemical energy difference between the sites which are separated by a translation up the $c$ axis. The strong Zener exchange between them will therefore act to immediately suppress the $A$-type magnetic structure.

\section{Conclusion}

In this article we have described the different process in doped ferrimagnets and antiferromagnets and highlighted the differences between them. We see that the low conductivity in the ferrimagnets is due to the small value of the intra-sublattice hopping energy as is clear from the low value of the intra-sublattice exchange.

Most strong superexchange interactions are antiferromagnetic. In the ferrimagnets the strong superexchange paths exist between the sites on different sublattices and charge transfer is inhibited between these sites because of there different chemical environment.

\section{Acknowledgments}

The author would like to pay especial thanks to Andrea Lehmann-Szweykow-. ska who introduced her to the problem of the garnets and has been so very helpful in discussions of this problem and also Ryszard Wojciechowski for collaborations on this problem. She has also benefited from many discussions on the manganites with Michael Zeise and Chatchai Srinitiwarawong and with Harry Blythe on magnetite. 


\section{References}

[1] P.G. de Gennes, Phys. Rev. 118, 141 (1960).

[2] Y. Nagaoka, Phys. Rev. 147, 392 (1966).

[3] K. Kubo, N. Ohata, J. Phys. Soc. Jpn. 33, 21 (1972); G.A. Gehring, D.J. Coombes, J. Magn. Magn. Mater. 117-181, 873 (1998).

[4] C. Zener, Phys. Rev. 82, 403 (1951).

[5] T.E. Mason, G. Aeppli, S.M. Haydon, A.P. Ramirez, H.A. Mook, Phys. Rev. Lett. 71, 919 (1993).

[6] J.M. Budnick, private communication.

[7] C.M. Varma, P.B. Littlewood, S. Schmitt-Rink, E. Abrahams, A.E. Ruckenstein, Phys. Rev. Lett. 63, 1996 (1989).

[8] J.M.D. Coey, M. Viret, S. von Molnar, Adv. Phys. 48, 167 (1999).

[9] A.P. Ramirez, J. Phys., Condens. Matter 9, 8171 (1997).

[10] S.P. Sena, R.A. Lindley, H.J. Blythe, Ch. Sauer, M. Al-Kafarji, G.A. Gehring, J. Magn. Magn. Mater. 176, 111 (1997).

[11] Z. Zhang, S. Satpathy, Phys. Rev. B 44, 13319 (1991).

[12] D. Ihle, B. Lorenz, J. Phys. C, Solid State Phys. 19, 5239 (1986).

[13] P.K. Larsen, R. Metselaar, J. Solid State Chem. 12, 253 (1975).

[14] A. Lehmann-Szweykowska, M.M. Kaczmarek, T. Lulek, in: Proc. 5th Int. School of Theoretical Physics, Zajaczkowo (Poland) 1998, Eds. T. Lulek, B. Lulek, A. Wal, World Scientific, Singapore 1999, p. 85.

[15] E. Pellegrin, L.H. Tjeng, F.M.F. de Groot, R. Hesper, G.A. Sawatzky, Y. Moritomo, Y. Tokura, J. Phys. IV (France) 7, 405 (1997).

[16] F.C. Zhang, T.M. Rice, Phys. Rev. B 37, 3759 (1988).

[17] A. Lehmann-Szweykowska, R.J. Wojciechowski, G.A. Gehring, Acta Phys. Pol. A, Part II of these Proceedings. 